\title{
Lyme Disease Presenting as Ankylosing Spondylitis: A Case Report
}

\author{
Megan Strauchman, Mark Morningstar \\ Natural Wellness \& Pain Relief Center, Grand Blanc, MI, USA \\ Email: ${ }^{\star}$ drmorningstar@nwprc.com
}

How to cite this paper: Strauchman, M. and Morningstar, M. (2020) Lyme Disease Presenting as Ankylosing Spondylitis: A Case Report. Case Reports in Clinical Medicine, 9, 354-359.

https://doi.org/10.4236/crcm.2020.911049

Received: October 18, 2020

Accepted: November 27, 2020

Published: November 30, 2020

Copyright $\odot 2020$ by author(s) and Scientific Research Publishing Inc. This work is licensed under the Creative Commons Attribution International License (CC BY 4.0).

http://creativecommons.org/licenses/by/4.0/

(c) (i) Open Access

\begin{abstract}
Lyme disease is a rapidly growing tick-borne infection in many parts of the United States. Due to the complexity of the various symptom presentations associated with Lyme disease, Borrelia burgdorferi, the bacteria that causes Lyme disease, is thought of as a disease-mimicking infection. The present case report summarizes the case of an adult female patient who was initially diagnosed with ankylosing spondylitis. After becoming refractory to autoimmune-based treatment interventions, she presented to an integrative medicine clinic where she was diagnosed with Lyme disease. Symptoms resolved after following and completing an anti-microbial based protocol, symptoms resolved. Her symptoms remained resolved at follow-up.
\end{abstract}

\section{Keywords}

Ankylosing Spondylitis, Infection, Lyme Disease

\section{Introduction}

The number of people diagnosed each year with Lyme disease is approximately 300,000 , making it the number one vector borne infection in the US [1]. While Lyme disease is a clinical diagnosis [2], symptom presentation can vary widely. Previous reports have found Lyme disease presenting as myocarditis [3], neuroretinitis [4], Bell's palsy [5], atrioventricular block [6], neuralgic amyotrophy [7], and sensorineural hearing loss [8].

Although there is debate about the most appropriate types and lengths of treatments for Lyme disease [9], the current guidelines recommend a 20-day course of antibiotics [2] or longer depending upon response to therapy. Despite this recommended therapy, some evidence [9] suggests that prolonged antibiotic therapy does not alter the natural course of the condition. Therefore, as other authors have suggested, alternative forms of therapy should be explored [10]. 
Ankylosing spondylitis (AS) is a chronic inflammatory disease that primarily affects the spine and its bony tendon attachments around the sacroiliac (SI) joint [11]. The cause of AS is still unknown. While it is historically classified as an autoimmune arthritide due to its association with HLA-B27, many patients with AS are seronegative [12]. Due to the variability in the presentation of AS symptoms, and hence the difficulty in its diagnosis, the Assessment of SpondyloArthritis International Society (ASAS) has developed criteria to aid in its classification [13]. These criteria can help identify patients with AS who are seronegative or seropositive.

The present case study provides an example of a patient who met the criteria for ankylosing spondylitis, and was also diagnosed with Lyme disease. A history of her symptoms, past medical treatments, and response to various treatments are described. The case report illustrates the concept that Lyme disease is often considered a disease mimicker. In the present care report, the symptoms were initially diagnosed as ankylosing spondylitis.

\section{Case Report}

A 55-year-old female school teacher reported to the author's (MNS) integrative medicine clinic for evaluation and management of chief complaints of pain in the left knee, right shoulder, and low back. She had been diagnosed 2 years earlier with ankylosing spondylitis by a rheumatologist. She was seropositive, and had the characteristic radiographic signs of sacroiliitis. The patient had a positive past medical history for the following concurrent diagnoses, primary hypothyroidism, cytomegalovirus infection, intestinal dysbiosis, malabsorption syndrome, and essential hypertension. Her resting blood pressure was 153/86. She had a positive family history of rheumatoid arthritis (sister).

At initial presentation, the patient was on the following medications: estradiol patch, $0.25 \mathrm{mg} / \mathrm{day}$; testosterone cream $6 \mathrm{mg} / \mathrm{mL}, 0.25 \mathrm{~mL}$ per day; progesterone, $150 \mathrm{mg}$ PO daily; naltrexone $5.5 \mathrm{mg}$ PO twice daily; lisinopril $10 \mathrm{mg}$ PO daily; Armor thyroid $75 \mathrm{mg}$ PO daily; and Enbrel, subcutaneous injection, $50 \mathrm{mg}$ once weekly. She was also taking the following supplements: vitamin D3 1000IU daily, ascorbic acid $500 \mathrm{mg}$ daily, multi-strain probiotic once daily, and liquid magnesium $250 \mathrm{mg}$ daily. These medications and supplements had been previously prescribed by an integrative medicine physician she had been seeing for the preceding 18 months. In addition, she was also placed on a paleo diet, limiting her carbohydrate intake to no more than 50 grams of net carbohydrates per day. Net carbohydrates are defined as the total carbohydrates minus any dietary fiber and sugar alcohols in the ingredients.

A review of previous laboratory results ordered by previous providers (rheumatology and integrative medicine) demonstrated the following significant results: positive HLA-B27, positive ANA screen with speckled pattern 1:320, multiple elevated repeat high sensitivity C-reactive protein (hsCRP) levels (5.5, 14.6, 9.9, 3.6, 15.1), elevated thyroid peroxidase antibodies (38), ALT 48, elevated 
thyroglobulin antibodies 4.1, elevated serum ferritin 277, hyperlipidemia, and low sodium 133. After the initial consultation, lab testing for Lyme disease was ordered. Patient received an ELISPOT Lyme disease test. This test measured $\mathrm{T}$ cell interferon gamma cytokine production following stimulation with Borrelia burgdorferi surface proteins. She had a positive result, and treatment for Lyme disease was initiated. The patient provided written consent to publish her non-identifying treatment information.

\section{Intervention and Outcome}

After her test came back positive, the following treatment was recommended at her next follow-up appointment: bovine colostrum-derived transfer factors (Transfer Factors PlasMyc, Researched Nutritionals) 1 capsule daily, a combination liquid herbal product (Blt\#1, Researched Nutritionals) 10 drops twice daily, dessicated adrenal glandular, 1 tablet three times daily, lactoferrin $300 \mathrm{mg}$ twice daily, and an over-the-counter desloratadine (Claritin ${ }^{\circledR}$ OTC, Bayer) 1 tablet daily. The patient declined to initiate antibiotic therapy.

After 30 days of this therapy, the patient reported having multiple "good days" days, where she "felt like her old self". She reported a consistent elevation in her resting blood pressure. Her blood pressure at follow-up was 143/100. At 22 days into this initial therapy, the patient increased her dose of Armor thyroid to 90 mg on her own. She stated that she had developed skin crawling sensations, and observed what she described as excess "mucus" in her stools. Back and shoulder pain remained unchanged.

At 60 days of therapy, the patient reported that her back and shoulder pain had markedly improved, decreasing from a constant frequency to predominantly an evening and nighttime occurrence. Her therapy was updated to include far-infrared sauna sessions 3 times weekly, and a trial of ultraviolet blood irradiation therapy (UVBI). This therapy consisted of daily treatments, 4 days weekly to 2.5 weeks, for a total of 10 treatments. The patient was re-assessed at 6 weeks after the $10^{\text {th }}$ treatment.

Lab findings at 6 weeks post-UVBI showed marked reductions in hsCRP (1.3), ALT 32, ferritin 44, negative ANA screen, and normal sodium 140. The patient reported a complete resolution in back and shoulder pain. Her resting blood pressure improved to $133 / 84$. She discontinued all supplements, as well as her Enbrel and naltrexone. The patient received an additional UVBI once monthly for the subsequent 3 months. The patient maintained her remaining prescriptions, as well as the once daily desloratadine. At that point, the patient was discharged from active care. At her final follow-up, 3 months after discharge, the patient was still pain-free, and her blood pressure was 134/81.

\section{Discussion}

The current case report details the history, treatment, and outcomes of a patient exhibiting symptoms of ankylosing spondylitis due to Lyme disease infection. 
Other case reports in the literature have detailed similar case reports in patients with autoimmune disorders. A case report by Briner and Habek [14] described how symptoms of multiple sclerosis can be caused by Lyme disease. Kolstoe and Messner have previously described how Lyme disease can mimic a variety of musculoskeletal autoimmune conditions, such as scleroderma, rheumatoid arthritis, juvenile rheumatoid arthritis, and myositis [15]. The current case study seems to be consistent with these previous studies showing a pattern of autoimmune musculoskeletal symptoms can be a manifestation of Lyme disease. Clinicians treating patients with these symptoms should consider the possibility of Lyme disease, especially when patients are refractory to autoimmune-based therapies.

Inflammatory arthritis symptoms are characteristic of late stage Lyme disease [16]. As in the current case, Lyme disease has been known to mimic other disease processes as well, including amyotrophic lateral sclerosis (ALS) [17], Guillain-Barre syndrome [17], lymphoma [18], as well as dental and facial pain disorders [19]. Therefore, it may be advisable to consider Lyme disease in cases like these where patients with complex neuromusculoskeletal diagnoses are refractory to standard treatment.

In the present case, the patient had received previous autoimmune-based therapies, such as low-dose naltrexone, initiating a paleo diet, and taking a biologic medication to reduce autoimmune-based inflammation. However, she did not experience any clinically significant benefit. The patient ultimately did not significantly respond to the nutrients initially prescribed by the author (MWM) until she received a trial of ultraviolet blood irradiation therapy (UVBI) in combination with the nutrient therapies. This therapy was extensively used in the 1940s and 1950s to treat many diseases including septicemia, pneumonia, tuberculosis, arthritis, asthma and even poliomyelitis [20]. However, despite its success, the lack of understanding of UVBI's mechanisms of action, as well as the achievements of antibiotics, have resulted in UVBI being eventually phased out of regular use since the 1950s [21].

Another therapy used in this study, desloratadine, has been shown to have anti-borrelia properties via its influence on the bacteria's manganese uptake [22]. However, it is unknown if or how this medication played a role in the patient's reported outcomes. Given the fact that this is a case study, limited conclusions can be made. It is unknown which of the therapies received contributed to the observed and reported outcomes, if any.

\section{Conclusion}

The present report describes the case of an adult female patient who was diagnosed with ankylosing spondylitis and later with Lyme disease. She was treated using a multimodal treatment approach. Following this treatment approach, the patient reported multiple subjective improvements in back and shoulder pain. Objective improvements in clinical laboratory values and vital signs were also 
observed. This study contributes to a body of previously published literature that describes the disease-mimicking nature of Borreliosis.

\section{Conflicts of Interest}

The authors declare no conflicts of interest regarding the publication of this paper.

\section{References}

[1] Centers for Disease Control and Prevention (2018) How Many People Get Lyme Disease? https://www.cdc.gov/lyme/stats/humancases.html

[2] Shor, S., Green, C., Szantyr, B., Phillips, S., Liegner, K., Burrascano Jr., J., Bransfield, R. and Maloney, E.L. (2019) Chronic Lyme Disease: An Evidence-Based Definition by the ILADS Working Group. Antibiotics, 8, 269. https://doi.org/10.3390/antibiotics8040269

[3] Gilson, J., Khalighi, K., Elmi, F., Krishnamurthy, M., Talebian, A. and Toor, R.S. (2017) Lyme Disease Presenting with Facial Palsy and Myocarditis Mimicking Myocardial Infarction. Journal of Community Hospital Internal Medicine Perspectives, 7, 363-365. https://doi.org/10.1080/20009666.2017.1396170

[4] Guliani, B.P., Kumar, S., Chawla, N. and Mehta, A. (2017) Neuroretinitis as Presenting and the Only Presentation of Lyme Disease: Diagnosis and Management. Indian Journal of Ophthalmology, 65, 250-252. https://doi.org/10.4103/ijo.IJO_151_17

[5] Cooper, L., Branagan-Harris, M., Tuson, R. and Nduka, C. (2017) Lyme Disease and Bell's Palsy: An Epidemiological Study of Diagnosis and Risk in England. British Journal of General Practice, 67, e329-e335. https://doi.org/10.3399/bjgp17X690497

[6] Bhattacharya, I.S., Dweck, M. and Francis, M. (2010) Lyme Carditis: A Reversible Cause of Complete Atrioventricular Block. Journal of the Royal College of Physicians of Edinburgh, 40, 121-122. https://doi.org/10.4997/JRCPE.2010.207

[7] Zhang, S., Zhang, L.Q., Wright, M. and Gater, D.R. (2017) Challenging Diagnosis and Inpatient Rehabilitation of Acute Bilateral Neuralgic Amyotrophy Possibly Attributed to Lyme Disease: A Case Report. $P M \& R, 10,770-774$.

https://doi.org/10.1016/j.pmrj.2017.11.018

[8] Peeters, N., van der Kolk, B.Y., Thijsen, S.F. and Colnot, D.R. (2013) Lyme Disease Associated with Sudden Sensorineural Hearing Loss: Case Report and Literature Review. Otology \& Neurotology, 34, 832-837. https://doi.org/10.1097/MAO.0b013e31827c9f93

[9] Borchers, A.T., Keen, C.L., Huntley, A.C. and Gershwin, M.E. (2015) Lyme Disease: A Rigorous Review of Diagnostic Criteria and Treatment. Journal of Autoimmunity, 57, 82-115. https://doi.org/10.1016/j.jaut.2014.09.004

[10] Lin, Y.P., Li, L.Y., Zhang, F.M. and Linhardt, R.J. (2017) Borrelia Burgdorferi Glycosaminoglycan-Binding Proteins: A Potential Target for New Therapeutics against Lyme Disease. Microbiology, 163, 1759-1766. https://doi.org/10.1099/mic.0.000571

[11] Kim, Y., Oh, H.C., Park, J.W., Kim, I.S., Kim, J.Y., Kim, K.C., et al. (2017) Diagnosis and Treatment of Inflammatory Joint Disease. Hip Pelvis, 29, 211-222.

https://doi.org/10.5371/hp.2017.29.4.211

[12] van der Linden, S. and van der Heijde, D. (2000) Clinical Aspects, Outcome Assessment, and Management of Ankylosing Spondylitis and Postenteric Reactive Arthritis. Current Opinion in Rheumatology, 12, 263-268. 
https://doi.org/10.1097/00002281-200007000-00005

[13] Raychaudhuri, S.P. and Deodhar, A. (2014) The Classification and Diagnostic Criteria of Ankylosing Spondylitis. Journal of Autoimmunity, 48-49, 128-133. https://doi.org/10.1016/j.jaut.2014.01.015

[14] Brinar, V.V. and Habek, M. (2010) Rare Infections Mimicking MS. Clinical Neurology and Neurosurgery, 112, 625-628. https://doi.org/10.1016/j.clineuro.2010.04.011

[15] Kolstoe, J. and Messner, R.P. (1989) Lyme Disease: Musculoskeletal Manifestations. Rheumatic Diseases Clinics of North America, 15, 649-656.

[16] Bitar, I. and Lally, E.V. (2008) Musculoskeletal Manifestations of Lyme Disease. Medicine \& Health Rhode Island, 91, 213-215.

[17] Burakgazi, A.Z. (2014) Lyme Disease-Induced Polyradiculopathy Mimicking Amyotrophic Lateral Sclerosis. International Journal of Neuroscience, 124, 859-862. https://doi.org/10.3109/00207454.2013.879582

[18] Bahrain, H., Laureno, R., Krishnan, J., Aggarwal, A. and Malkovska, V. (2007) Lyme Disease Mimicking Central Nervous System Lymphoma. Cancer Investigation, 25, 336-339. https://doi.org/10.1080/07357900701357977

[19] Heir, G.M. (1997) Differentiation of Orofacial Pain related to Lyme Disease from Other Dental and Facial Pain Disorders. Dental Clinics of North America, 41, 243-258.

[20] Hamblin, M.R. (2017) Ultraviolet Irradiation of Blood: “The Cure That Time Forgot"? Advances in Experimental Medicine and Biology, 996, 295-309. https://doi.org/10.1007/978-3-319-56017-5_25

[21] Boretti, A., Banik, B. and Castelletto, S. (2020) Use of Ultraviolet Blood Irradiation Against Viral Infections. Clinical Reviews in Allergy \& Immunology, 7, 1-12. https://doi.org/10.1007/s12016-020-08811-8

[22] Wagh, D., Pothineni, V.R., Inayathullah, M., Liu, S., Kim, K.M. and Rajadas, J. (2015) Borreliacidal Activity of Borrelia Metal Transporter A (BmtA) Binding Small Molecules by Manganese Transport Inhibition. Drug Design, Development and Therapy, 9, 805-816. https://doi.org/10.2147/DDDT.S77063 